\title{
Cryptogenic strokes and embolic strokes of unknown source: lights and shadows of a new construct
}

\author{
Marco Masina \\ Complex Unit, Geriatric ward and Stroke Care Area, Bentivoglio Hospital, AUSL Bologna, Italy
}

\section{Introduction}

The term cryptogenic (i.e., undetermined) stroke (CS) defines a group of strokes of unknown etiology. The characteristics of CS vary according to three different definitions available: the Trial of $\mathrm{Org} 10172$ in acute stroke treatment (TOAST) criteria, ${ }^{1}$ the Causative classification of stroke system (CCS), ${ }^{2}$ and the Atherosclerosis, small vessel disease, cardiac causes, and other uncommon causes (ASCO). ${ }^{3}$ Each definition has pros and cons. According to TOAST definition, cryptogenic strokes include both cases with multiple potential etiologies and/or with incomplete diagnostic work-up. It is considered to overestimate the number of all strokes by up to $40 \%{ }^{4,5}$ The CCS and the ASCO criteria are more restrictive and cryptogenic strokes account for about $25-30 \%$ of all strokes. ${ }^{5}$ The CCS criteria differentiate cryptogenic strokes in two subtypes: cryptogenic embolism and other cryptogenic. The CCS assigns patients accordingly to the most likely mechanism. The ASCO classification is a complex system encompassing 625 phenotipic subtypes of stroke and requires an extensive collection of data.

Correspondence: Marco Masina, Complex Unit, Geriatric ward and Stroke Care Area, Bentivoglio Hospital, AUSL Bologna, via Marconi 35, 40010 Bentivoglio (BO), Italy. Tel.: +39.051.6644394.

E-mail: marco.masina@ausl.bologna.it

Key words: Embolic stroke of undetermined source; cardioembolic stroke; minor embolic source.

Conflict of interest: the author declares no potential conflict of interest.

Received for publication: 6 April 2016.

Accepted for publication: 6 April 2016

This work is licensed under a Creative Commons Attribution NonCommercial 4.0 License (CC BY-NC 4.0).

CCopyright M. Masina, 2016

Licensee PAGEPress, Italy

Italian Journal of Medicine 2016; 10:171-174

doi:10.4081/itjm.2016.720
Recently the new construct of Embolic stroke of unknown source (ESUS) has been proposed by the CS/ESUS International Working Group in order to define the subgroup of cryptogenic strokes in which specific mechanisms of stroke have been excluded and evidence of covert paroxysmal atrial fibrillation (AF) or minor cardiogenic embolic conditions, atheroembolism, cancer associated and paradoxical embolism through a patent foramen ovale (PFO) or pulmonary fistula is found. ${ }^{6}$ The prevalence of ESUS is still debated. In the Athens Registry ESUS accounts for $1 / 3$ of cryptogenic strokes and represents about $11 \%$ of all strokes. ${ }^{7}$ The ESUS Global Registry which surveyed 19 stroke research centers in 19 different countries reported a prevalence of $16 \%$ but an additional $14 \%$ had an incomplete evaluation. ${ }^{8}$

The challenge posed by cryptogenic strokes is both clinical and economical.

Main guidelines [by the American Heart Association/American Stroke Association (ASA/AHA) and the European Stroke Organization (ESO)] do not recommend an extensive diagnostic work-up and many strokes result cryptogenic because the search was actually incomplete. ${ }^{9,10}$ Echocardiogram (transthoracic and/or transesophageal), prolonged hearth rhythm monitoring, intracranial echo-Doppler, intra and extracranial angio-computed tomography or magnetic resonance imaging including aortic arch, and laboratory evaluation of rare hypercoagulability causes are not currently recommended in a routine diagnostic work-up. In Tables 1 and 2 of their review, Stornello et al. ${ }^{11}$ report a complete list of genetic, traumatic, drug-related, inflammatory, and autoimmune diseases which are all potentially responsible for a stroke of rare etiology. A widespread searching program of all those causes in all patients would improve diagnostic precision but it would also make costs unsustainable. The diagnosis of cervical arterial dissection, pulmunary fistula, antiphospholipid syndrome, genetic thrombophilic diseases, Fabry disease, and occult cancer should not be missed but should be guided by clinical features or anamnestic history. ${ }^{5}$ Recently an elevated level of D-dimers has been suggested as a useful biomarker leading to the search of occult cancer 
but it requires further investigation. ${ }^{12}$ To date the decision to perform the diagnostic work-out aimed to rare causes is up to the clinical judgment of stroke physicians.

\section{Embolic stroke of unknown source}

Letting apart the search for rare causes, the extensive search of embolic sources in CS is more appealing both because of the availability of diagnostic devices and the potential effectiveness of new anticoagulant therapies. The ESUS construct considers the potential of a number of minor-risk cardioembolic sources, as reported by Stornello et al. ${ }^{11}$ The long list contains structural cardiopathies (mitral valve myxomatous valvulopathy with prolapse, mitral annular calcification, aortic valve stenosis, aortic valve calcification, atrial appendage stasis with reduced flow velocities or spontaneous echodensities, atrial septal aneurysm, Chiari network, left ventricular moderate systolic or diastolic dysfunction both global or regional, left ventricular noncompaction, endomyocardial fibrosis), together with arrhythmic conditions as atrial asystole and sick-sinus syndrome, atrial flutter, and atrial high-rate episodes. ${ }^{6}$

The spotlight is on covert AF or other embolic arrhythmias because they are frequently found in the follow-up of cryptogenic strokes. Since new long term implantable monitoring devices have become available the prevalcence of AF in stroke has raised. However many AF are short (less than $30 \mathrm{~s}$ ) or occur months or years after the stroke event, raising the question of their significance for an embolic source. ${ }^{13-16}$ The relationship between $\mathrm{AF}$ and stroke seems more complex than the simple mechanistic model of a diysrythmia related stasis of blood followed by thrombus formation and embolism. The risk of stroke increases shortly after the onset of AF well before AF can induce structural remodelling of the atrium. ${ }^{17}$ On the other hand about one third of patients with AF and stroke shows AF after (not before) the ischemic event. ${ }^{18}$ Recently Kamel has proposed a new model in which a structural remodelling of the atrium (atrial cardiopathy) related to traditional vascular risk factors might play a role in thrombogenesis analogous to that of ventricular cardiopathy. ${ }^{21}$ Atrial cardiopathy may precede and/or cause AF. When both conditions are established, the risk of thromboembolism increases further. That new model has yet to be confirmed, but if it does, echocardiogram or other cardiac imaging will become mandatory.

Patent foramen ovale represents an often overestimated potentially embolic condition especially in young patients. In his complete review Donti critically reports that, given the high prevalence of PFO in the general population, an association between $\mathrm{PFO}$ and CS in the same patient does not necessarily imply a causal link to the etiology of the stroke. ${ }^{21,21} \mathrm{PFO}$ is often a bystander and it is actually not responsible of the acute event. Moreover stroke recurrences in patient with PFO are rare. ${ }^{22}$ Overall, results of recent trials on PFO closure have been inconclusive and complications not irrilevant. Meta-analysis showed a trend towards a superiority of PFO closure but the quality of the evidence is poor. The event reduction with closure seems to be low, and the number needed to treat high. ${ }^{23}$ Donti concludes that transcatheter closure of the PFO is a valid option but, while we wait for more solid data about its efficacy and safety as compared to medical therapy, it should be offered only to carefully selected patients more likely to have a causal PFO. The risk of paradoxical embolism (RoPE) score may guide individualized and effective patient selection for PFO closure. ${ }^{20}$

\section{Embolic stroke of unknown source recurrences rate}

The diagnosis of structural cardiac and valve conditions related to ESUS requires an echocardiogram (transthoracic and/or transesophageal), which is still not recommended by guidelines in the routine diagnostic work-up. That explains why retrospective studies on ESUS are scarce and the prevalence and recurrence rate of ESUS are still uncertain. In his two recent papers, Ntaios ${ }^{7,24}$ reported a large cohort of patients from the Athens Registry in which the prevalence of ESUS was about $11 \%$ of all strokes and the rate of stroke recurrence at 1 year was $11.3 \%$. But those findings have been debated and other population or hospital-based registry studies reported a 3-9\% recurrence rate during the first year. ${ }^{25-27}$

Our retrospective analysis is limited to a small cohort of a single Stroke Unit in which transthoracic echocardiogram was routinely performed and reports a stroke recurrence rate at 1 year in ESUS of $4.4 \%$. Interestingly that doubles that of CS and is the same as cardioembolic strokes suggesting a potential relation to embolism.

\section{How embolic stroke of unknown source construct may affect secondary prevention strategy}

To date no recommendation for secondary prevention in cryptogenic stroke is available and antiplatelet agents are usually prescribed without secure evidence ${ }^{8} 10$ The use of warfarin in minor risk embolic sources has been discouraged by hemorrhagic events. ${ }^{28}$ Being a consistent fraction of cryptogenic stroke associated with a potentially embolic source, the availability of new oral anti coagulant (NOAC) 
drugs in secondary prevention has raised interest because of a potentially net benefit in balancing prevention of ischemic recurrences and hemorrhagic events.

The new ESUS construct has lead to randomised clinical trials comparing aspirin versus NOACs in secondary prevention. The RE-SPECT ESUS trial comparing aspirin and dabigatran and the NAVIGATE ESUS trial comparing aspirin to rivaroxaban are already ongoing. ${ }^{29,30}$ The Atticus study comparing aspirin to apixaban has not enrolled patients yet. ${ }^{31}$

Unfortunately ESUS is a clinical construct that includes a range of different conditions. It is not surprising that criteria of patient selection differ among studies. For example heart rate monitoring is required by all studies, but while RE-SPECT ESUS includes patients in which an AF shorter than 6 min in a $20 \mathrm{~h}$ monitoring has been detected, NAVIGATE ESUS excludes all patients with AF detected in a $24 \mathrm{~h}$ monitoring. Other differences in selection criteria are in the inclusion of different major- and minor-risk cardioembolic sources and in neuroimaging.

Stroke physicians are challenged by the intent of decreasing the number of cryptogenic strokes, by the need of avoiding the high costs of extensive diagnostic search and by the uncertain benefit of drugs in secondary prevention of recurrences. As a new costruct, ESUS proposes a larger but sustainable minimum set of diagnostic exams to be carried out in all stroke patients. ESUS criteria might be helpful in confirming the advantage of anticoagulation with NOACs in some CS with minor-risk embolic sources but, because of the heterogenity of the embolic sources, the net benefit of NOACs might be limited only to some and not to all conditions.

\section{References}

1. Adams HP Jr, Bendixen BH, Kappelle LJ, et al. Classification of subtype of acute ischemic stroke. Definitions for use in a multicenter clinical trial. TOAST. Trial of Org 10172 in Acute Stroke Treatment. Stroke 1993;24:35-41.

2. Ay H, Benner T, Arsava EM et al. A computerized algorithm for etiologic classification of ischemic stroke : the Causative Classification of Stroke System. Stroke 2007;38:2979-84.

3. Amarenco P, Bogousslavsky J, Caplan LR, et al. New approach to stroke subtyping: the A-S-C-O (phenotypic) classification of stroke. Cerebrovasc Dis 2009;27:502-8.

4. Marnane M, Duggan CA, Sheehan OC, et al. Stroke subtype classification to mechanism-specific and undetermined categories by TOAST, A-S-C-O, and causative classification system: direct comparison in the North Dublin population stroke study. Stroke 2010;41:1579-86.

5. Fonseca AC, Ferro JM. Cryptogenic stroke. Eur J Neurol 2015;22:618-23.

6. Hart RG, Diener H-C, Coutts SB, et al. Embolic strokes of undetermined source: the case for a new clinical construct. Lancet Neurol 2014;13:429-38.
7. Ntaios G, Papavasileiou V, Milionis H, et al. Embolic strokes of undetermined source in the Athens stroke registry: a descriptive analysis. Stroke 2015;46:176-81.

8. Perera KS, Vannasche T, Bosch J, et al. Embolic strokes of undetermined source: Prevalence and patient features in the ESUS Global Registry. Int J Stroke 2016;11:526-33.

9. Kernan WN, Ovbiagele B, Black HR, et al. Guidelines for the prevention of stroke in patients with stroke and transient ischemic attack: a guideline for healthcare professionals from the American Heart Association/American Stroke Association. Stroke 2014;45:2160-236.

10. European Stroke Organization (ESO) Executive Committee; ESO Writing Committee. Guidelines for the management of ischaemic stroke and transient ischaemic attack. Cerebrovasc Dis 2008;25:457-507.

11. Stornello M, Cappellani R, Micieli G, et al. Cryptogenic stroke. Ital J Med 2016;10:185-94.

12. Kim SJ, Park JH, Lee MJ, et al. Clues to occult cancer in patients with ischemic stroke. PLoS One 2012;7:e44959.

13. Rabinstein AA, Prolonged cardiac monitoring for detection of paroxysmal atrial fibrillation after cerebral ischemia. Stroke 2014;45:1208-14.

14. Gladstone DJ, Spring M, Dorian P, et al. Atrial fibrillation in patients with cryptogenic stroke. N Eng J Med 2014;370:2467-77.

15. Sanna T, Diener HC, Passman RS, et al. Cryptogenic stroke and underlying atrial fibrillation. N Eng J Med 2014;370:2478-86.

16. Sposato LA, Cipriano LE, Riccio PM, et al. Very short paroxysms account for more than half of the cases of atrial fibrillation detected after stroke and TIA: a systematic review and meta-analysis. Int J Stroke 2015;10:801-7.

17. Healey JS, Connolly SJ, Gold MR, et al. Subclinical atrial brillation and the risk of stroke. N Engl J Med 2012;366:120-9.

18. Brambatti M, Connolly SJ, Gold MR, et al. Temporal relationship between subclinical atrial brillation and embolic events. Circulation 2014;129:2094-9.

19. Kamel H, Okin PM, Elkind MSV, Iadecola C. Atrial fibillation and mechanisms of stroke. Time for a new model. Stroke 2016;47:895-900.

20. Donti A. Patent foramen ovale and ischemic stroke: more shadows than lights? What the internist should know. Ital J Med 2016;10:175-84.

21. Kent DM, Ruthazer R, Weimar C, et al. An index to identify stroke-related vs incidental patent foramen ovale in cryptogenic stroke. Neurology 2013;81:619-25.

22. Mas JL, Arquizan C, Lamy C, et al. Recurrent cerebrovascular events associated with patent foramen ovale, atrial septal aneurysm, or both. N Engl J Med 2001;345:1740-6.

23. Li J, Liu J, Liu M, et al. Closure versus medical therapy for preventing recurrent stroke in patients with patent foramen ovale and a history of cryptogenic stroke or transient ischemic attack. Cochrane Database Syst Rev 2015;9:CD009938.

24. Ntaios G, Papavasileiou V, Milionis H, et al. Embolic strokes of undetermined source in the Athens stroke registry: an outcome analysis. Stroke 2015;46:2087-93.

25. Li L, Yiin GS, Geraghty OC, et al. Incidence, outcome, risk factors, and long-term prognosis of cryptogenic transient ischaemic attack and ischaemic stroke: a population-based study. Lancet Neurol 2015;14:903-13. 
26. Mahagne MH, Lachaud S, Suissa L. Letter by Mahagne et al. regarding article, 'Embolic strokes of undetermined source in the Athens stroke registry: a descriptive analysis'. Stroke 2015;46:e69.

27. Putaala J, Nieminen T, Haapaniemi E, et al. Undetermined stroke with an embolic pattern - a common phenotype with high early recurrence risk. Ann Med 2015;47:406-13.

28. Sacco RL, Prabhakaran S, Thompson JL, et al. Comparison of warfarin versus aspirin for the prevention of recurrent stroke or death: subgroup analyses from the Warfarin-Aspirin Recurrent Stroke Study. Cerebrovasc Dis 2006;22:4-12.

29. Boehringer Ingelheim. Dabigatran etexilate for second- ary stroke prevention in patients with embolic stroke of undetermined source (RE-SPECT ESUS). ClinicalTrials.gov identifier: NCT02239120. Available from: https://clinicaltrials.gov/ct2/show/NCT02239120 Accessed: 01/01/2016.

30. Population Health Research Institute. Stroke and cognition: ESUS Registry, IMPACT, NAVIGATE ESUS. Available from: http://www.phri.ca/research/stroke-cognition/navigate-esus-111/ Accessed: 01/01/2016.

31. Geisler T, Ziemann U. Apixaban for treatment of embolic stroke of undetermined source (ATTICUS). ClinicalTrials.gov identifier: NCT02427126. Available from: https://clinicaltrials.gov/ct2/show/NCT02427126 Accessed: 01/01/2016. 